\title{
Valoración de la información sobre el trasplante renal que reciben los pacientes en los centros periféricos de hemodiálisis
}

\author{
Cirera Segura, Francisco \\ Reina Neyra, Macarena
}

Club de Diálisis. Hospitales Universitarios

Virgen del Rocío. Sevilla

\section{RESUMEN}

La educación sanitaria al paciente renal es una prioridad para la enfermería nefrológica, ya que la adecuada información y preparación psicológica es indispensable para que el paciente y su familia sean capaces de adaptarse a los cambios de vida que suponen la diálisis y posteriormente el trasplante.

Nuestro objetivo es determinar los conocimientos del personal de enfermería sobre el trasplante, en los centros periféricos y valorar de qué manera se da la información a los pacientes.

Es un estudio descriptivo que se ha llevado a cabo en 9 centros periféricos de hemodiálisis en la provincia de Sevilla. Para ello se han elaborado unas encuestas dirigidas al personal de enfermería, que constan de un total de 21 preguntas, formadas por un total de 113 ítems, en las que se valoran aspectos como la forma de dar la información al paciente, y los conocimientos en tres apartados como son la lista de espera, el proceso del trasplante y los autocuidados tras el mismo.

Han contestado a la encuesta un $62 \%$ de la población. De los resultados cabe destacar que menos del $50 \%$ de los pacientes solicitan información sobre el trasplante. Un 37\% de las encuestadas afirman disponer de un protocolo de información, destaca la calidad de la información acerca de la etapa del trasplante y de los autocuidados tras el mismo.

Correspondencia:
Francisco Cirera Segura.
C/ Ángel Ripoll Pastor, No $4-5^{\circ}$ A.
41006 Sevilla.
e-mail: $f$ cirera@hotmail.com

Concluimos que la mayor parte del personal de enfermería de los centros periféricos tiene amplia información sobre el trasplante renal, aunque en algunos aspectos, esta información debería completarse o actualizarse.

$\begin{array}{ll}\text { PALABRAS CLAVE: } & \text { TRASPLANTE RENAL } \\ & \text { EDUCACIÓN SANITARIA } \\ & \text { AUTOCUIDADOS }\end{array}$

EVALUATION OF THE INFORMATION ON KIDNEY TRANSPLANTS RECEIVED BY PATIENTS IN PERIPHERAL HAEMODIALYSIS UNITS

\section{SUMMARY}

Health education in kidney patients is a priority for nephrology nursing, as the right information and psychological preparation is essential for the patient and their family to be able to adapt to the life changes that dialysis and transplant involve.

Our objective is to determine the knowledge of nursing personnel on transplants in the peripheral units and to evaluate in what way the information is given to patients.

This is a descriptive study performed in 9 peripheral haemodialysis centres in the province of Seville. To carry it out, surveys were drawn up for the nursing staff with a total 21 questions formed by 113 items, where aspects were evaluated such as the form of giving the patient information and knowledge in three sections: the waiting list, the transplant process and later self-care. 
Sixty-two percent of the population answered the survey. Among the results, we must highlight that under $50 \%$ of patients ask for information on the transplant. Thirty-seven percent of those surveyed said that they had an information protocol and stressed the quality of the information on the transplant stage and later self-care.

We conclude that the large part of nursing personnel in the peripheral units have broad information on kidney transplants, although in some aspects this information should be completed or updated.

KEYWORDS: KIDNEY TRANSPLANT

HEALTH EDUCATION

SELF-CARE

\section{INTRODUCCIÓN}

La educación sanitaria al paciente renal ha sido siempre una prioridad para los profesionales de la salud. Actualmente, aumenta cada vez más esta responsabilidad fundamental en el proceso de enfermería y en concreto, en la enfermería nefrológica, es una actividad imprescindible durante los tres periodos por los que puede pasar el paciente renal, como son la etapa pre-diálisis, la hemodiálisis o diálisis peritoneal y el trasplante, ya que la adecuada información y preparación psicológica son imprescindibles para que el paciente y su familia sean capaces de aceptar la nueva situación y adaptarse a los cambios de vida que esto representa ${ }^{(1)}$.

Esta educación pretende lograr la máxima independencia del paciente, conseguir una buena rehabilitación $e$ integración social y laboral y promover su capacidad en la toma de decisiones en la medida de lo posible.

El trasplante renal representa el tratamiento de elección para estos pacientes ya que comporta el restablecimiento de la función renal con recuperación de alteraciones clínicas, una liberación con respecto a las sesiones de hemodiálisis y por tanto un mejor equilibrio físico y psíquico del paciente $e^{(1)}$.

Tras el trasplante, por tanto, se restablece la calidad de vida de los pacientes en diálisis, que había disminuido, con respecto a la población general ${ }^{(2)}$ y es superior a la de aquellos que se mantienen en tratamiento dialítico ${ }^{(3)}$. Pero como toda opción terapéutica, el trasplante renal no está exento de limitaciones y complicaciones asocia- das ${ }^{(3)}$. Además implica unos cuidados que tanto el paciente, la familia, y el personal de enfermería, deberían conocer y valorar antes de que llegue el momento de realizar dicho trasplante.

Si estos beneficios y complicaciones son conocidos y asimilados antes de ese momento, el estrés y la ansiedad que presentan estos pacientes, se verían disminuidos ya que conocerían los procedimientos a los que van a ser sometidos antes, durante y después del trasplante renal ${ }^{(4)}$. Además, la toma de la decisión de trasplantarse o no, se haría basándose en una información veraz y no sobre una serie de creencias o informaciones incorrectas recibidas a través de otros pacientes o personal no sanitario, lo cual puede crear un desconcierto en el paciente en el momento del trasplante, que implica una carga de ansiedad añadida a la del procedimiento en si.

Es necesario dar a conocer que autocuidados deberá realizar el paciente trasplantado antes de que el trasplante. Por esto, creemos importante conocer la información global que se proporciona a los pacientes en los centros periféricos de hemodiálisis sobre este tema. Esta información, que debemos tener los profesionales dedicados a trabajar con enfermos tributarios de un trasplante, no siempre es tan completa como se debiera esperar por diferentes razones ${ }^{(5)}$, por lo que nuestro objetivo es hacer un estudio sobre la información que el personal de enfermería de los centros periféricos posee acerca del trasplante renal y como la transmiten a los pacientes.

Un objetivo posterior, sería continuar este trabajo con la redacción de un protocolo de información y la organización de sesiones formativas para acercar el proceso de trasplante al personal de enfermería de los centros periféricos de hemodiálisis, que no tienen las facilidades para acceder a un proceso que se lleva acabo de forma hospitalaria.

\section{MATERIAL Y MÉTODOS}

Se trata de un estudio descriptivo cuya muestra coincide con la población y la constituyen los 93 sujetos que forman el personal de enfermería de los 9 centros periféricos de Sevilla capital, incluyendo los dos centros periféricos de hemodiálisis de los hospitales de referencia.

Para la realización del estudio se han diseñado unas encuestas dividas en cuatro bloques, con preguntas que contienen varios ítems (Anexo 1):

El primer bloque (preguntas 1-5) contiene 21 ítems y recoge la forma en que cada centro informa al paciente sobre el trasplante renal. 
El segundo bloque (preguntas 6-11) contiene 36 ítems sobre los procedimientos que se le realizarán al paciente para entrar en la lista de espera así como la información sobre el pre trasplante inmediato.

En el tercer bloque (preguntas 12-8) contiene 38 ítems y valora la información que se le da al paciente sobre el acto en si del trasplante renal, y su estancia en la unidad de trasplante.

El cuarto y último bloque (preguntas 19-21) contiene 18 ítems y evalúa los cuidados y medidas que tendrá que conocer el paciente una vez que el trasplante ha tenido lugar.
Las encuestas se dejaron en los centros durante un periodo de una a dos semanas, y se solicitó a los encuestados/as que las contestaran de manera individual sin consultar al resto del personal del centro o de fuera del mismo, para asegurar que la información recibida se corresponde con la que cada enfermero/a proporciona al paciente.

Para analizar los datos obtenidos se ha utilizado el paquete estadístico SPSS 11.0, realizando tablas de distribución de frecuencias y porcentajes, representaciones gráficas, y por último, se han interpretado los resultados obtenidos y comentado los hallazgos.

\section{BLOQUE 1}

1. ¿Qué porcentaje de pacientes demandan información sobre el trasplante renal al personal?

a. $100 \%$ de los tributarios a trasplante renal.

b. $75 \%$ de los tributarios a trasplante renal.

c. $50 \%$ de los tributarios a trasplante renal.

d. Menos del $50 \%$ de los tributarios a trasplante renal.

2. ¿Existe algún protocolo en el centro donde trabajas acerca del trasplante renal?

a. Si, pero no lo usamos.

b. Si, pero no lo conozco.

c. No existe.

d. Existe personal espcífico dentro del equipo de salud encargado del control de los procedimientos.

e. En caso de respuesta afirmativa indica que protocolo se utiliza.

3. La información sobre el trasplante se da:

a. Porque la pide el paciente.

b. De manera espontánea por el personal.

c. De forma programada según un protocolo

d. Se hace una encuesta inicial para detectar las necesidades del paciente.

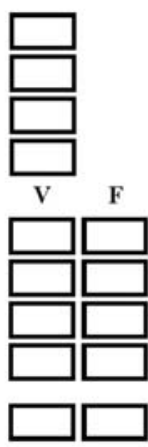

4. Cuando informamos al paciente sobre el trasplante renal:

a. Se incluye a la familia en la educación sobre el trasplante

b. Lo hacemos durante la sesión de hemodiálisis exclusivamente con el paciente.

c. Lo hacemos en un lugar destinado por el centro para impartir cursos o informar a los familiares.

d. Se les proporciona información escrita como complemento a la verbal.

5. ¿A qué tema dan más importancia y por lo tanto, piden más información los pacientes? Ordénalos por orden e importancia

a. Autocuidados post trasplante

b. Intervención quirúrgica.

c. Preparación para el trasplante.

d. Lista de espera.

e. Dietas tras el trasplante.

f. Medicación.

g. Otros

Cuales
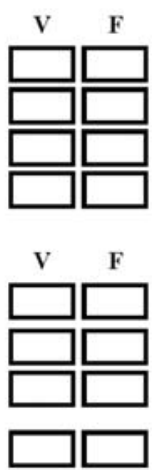

\section{BLOQUE 2}

6. Previo a la inclusión en la lista de espera necesitamos hacer las siguientes determinaciones:

a. Hematologia.

b. Bioquimica.

c. Serología.

d. Otras

Indicar cual

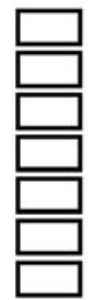

V F

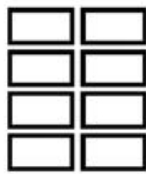


7. Si te encuentras en tu centro con los siguientes pacientes, ¿cuáles de ellos podría trasplantarse?

a. Paciente con V.I.H.

b. Paciente con hemorragia digestiva.

C. Paciente fumador con patología pulmonar severa.

d. Paciente con neoplasia con mas de 5 años sin recidiva.

e. Paciente con obesidad importante.

f. Paciente psicótico no controlado.

g. Paciente consumidor activo de drogas y alcohol.

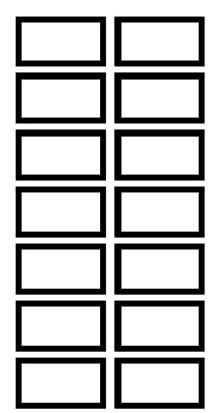

8. Sobre los procedimientos a llevar a cabo por el centro antes de la inclusión en la lista $\quad$ V $\quad F$ de espera:

a. Se puede incluir en lista de trasplante a la espera de los Ac Citotóxicos.

b. Se debe llevar un control de las contraindicaciones para el trasplante junto con un informe actualizado de cada paciente.

C. El paciente debe firmar un consentimiento informado.

d. Se deben tener una serie de pruebas complementarias como enema opaco, pruebas cardiológicas y ginecológicas.

e. De forma excepcional se solicitan una cistografía funcional y una tomografía renal.

f. Se debe informar al paciente de que una vez en la lista tiene la obligación de trasplantarse

9. Si un paciente te pide información acerca del Cross-Match, le podrías decir que:

a. Es una prueba de inmunología.

b. Es una prueba bioquímica.

C. Su resultado no es determinante para el trasplante.

d. Se utilizan células de ganglios linfáticos y bazo del donante.

e. Se utiliza sangre del receptor.

10. Cuando un paciente te pregunta acerca de los Ac. Citotóxicos:

a. Han de sacarse 30 días después de cualquier transfusión.

b. Hay que comprobar la recepción del tipaje, el archivo y el registro.

C. Se deben sacar cada 30 días para remitirlos al centro correspondiente

d. Han de sacarse tras una trasplantectomía.

e. Las únicas vías para su desarrollo son las trasfusiones y la trasplantectomía.

f. Son anticuerpos desarrollados por el paciente frente a antígenos del injerto.

11. Debes informar al paciente, para reducir su ansiedad, que en el pre-trasplante inmediato le harán:

a. Hemodiálisis.

b. Placas de tórax y abdomen.

C. Electroencefalograma.

d. Canalización catéter central solo si no es posible la canalización periférica.

e. Comprobación de la vejiga y uréteres mediante cistouretrografía miccional seriada (CUMS).

f. Urocultivo, esputos y frotis nasal.

g. No se administrará medicación inmunosupresora y antibióticos hasta el post-trasplante.

h. Otros

Cuales
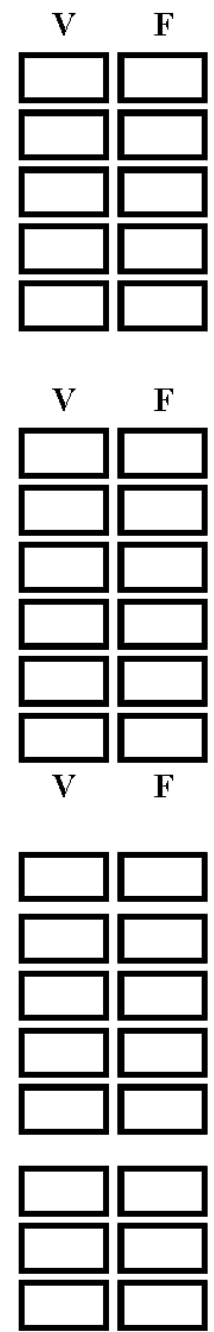


\section{BLOQUE 3}

12. Sobre el protocolo hospitalario del trasplante renal, le podría contar que:

a. El paciente puede permanecer en la UCI un tiempo mínimo de 24 horas.

b. El aislamiento en la unidad de trasplante se mantendrá hasta el alta del paciente.

C. La intervención quirúrgica suele durar una hora y media.

d. Es imprescindible el uso de batas, gorros, mascarillas y papis por los familiares durante el tiempo de aislamiento.

e. Serán seleccionados de la lista de espera de 5 a 7 posibles receptores.

13. ¿Cuales son las complicaciones quirúrgicas más frecuentes del trasplante renal?

a. Obstrucción de la vía urinaria

b. Fístula urinaria.

C. Estenosis de la arteria renal o trombosis venosa.

d. Infecciones transmisibles por el injerto.

e. Rotura renal.

14. Si el paciente te preguntara la complicación médica más frecuente tras el trasplante, tu le diría que es:
a. Anemia.
b. Poliglobulia.
C. Hipertensión arterial.
d. Diabetes esteroidea.
e. Altos niveles de P.T.H.

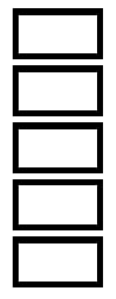

15. Sobre el nuevo riñón:

a. La zona más frecuente es la colocación del riñón en fosa iliaca o fosa lumbar.

b. En España todavía no existe la posibilidad de donante vivo.

C. Es posible la transmisión al receptor de enfermedades a través del injerto.

d. La herida quirúrgica no requiere cuidados específicos al ser de poca longitud.

e. Es imprescindible la extracción previa del riñón del receptor para proceder al trasplante renal.

f. El tiempo de vida media del injerto suele ser de 20 años.

g. El paciente solo se puede trasplantar 2 veces.

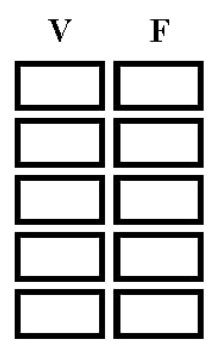

16. ¿Que pruebas complem entarias o procedimientos podría necesitar tras el trasplante renal?

a. Eco-Doppler o Ecografía simple.

b. Biopsia renal.

c. Arteriografía.

d. Exámenes radiológicos, e isotópicos.

e. Pielografía anterógrada.

f. Hemodiálisis. 
17. Ante la pregunta de qué ocurrirá con el acceso vascular tras el trasplante:

a. La F.A.V.I. se conserva mientras no presente complicaciones.

b. El catéter de diálisis peritoneal se extrae mediante intervención quirúrgica.

C. Se deben mantener los cuidados de la F.A.V.I. que permanezca activa.

d. Se suprime la F.A.V.I al año del trasplante.

e. Se mantiene el catéter de diálisis peritoneal pero no los de hemodiálisis.

18. ¿Ante cuál de estos síntomas sospecharías de rechazo agudo del injerto renal?

a. En la mesa de quirófano observas que el parénquima renal queda moteado.

b. Eosinofilia.

C. Se produce una disminución de la diuresis y aparece deterioro de la función renal.

d. Debemos sospechar de rechazo agudo solo si la fiebre es superior a $38,5^{\circ} \mathrm{C}$

e. Aumento de peso y de la tensión arterial.

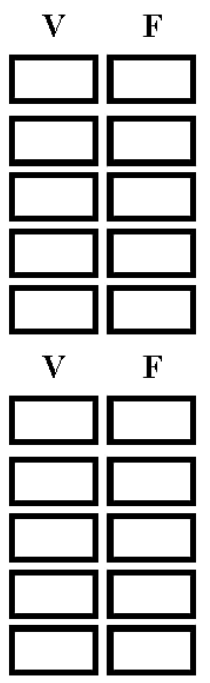

\section{BLOQUE 4}

19. La información que se le debe dar a los pacientes acerca de los inmunosupresores debe incluir:

a. Que la dosis a utilizar disminuirá con el tiempo .

b. Se deberán tomar solo durante los tres años siguientes al trasplante.

C. Se administrará tras la primera ingesta del día.

d. Los efectos secundarios pueden incluir desde acné e hirsutismo a enfermedades neoplásicas.

e. No tienen efecto acumulativo en el tiempo.

20. En pacientes en edad de gestación se les debería informar:

a. Es preferible el embarazo en el periodo de diálisis que tras el trasplante.

b. En la mujer trasplantada la menstruación y la ovulación pueden reanudarse.

c. La disfunción eréctil en los varones se recupera en aproximadamente $60 \%$ de los casos

d. No se recomienda dar el pecho a las mujeres tratadas con Ciclosporinas.

e. El trasplante renal mejora sensiblemente las disfunciones sexuales.

f. El embarazo es habitualmente más corto que en el resto de las mujeres.

g. Debe evitarse el embarazo antes del primer año de trasplante.

21. Sobre los autocuidados tras el trasplante debemos informar al paciente de:

a. Es necesario abandonar hábitos nocivos como el tabaco y el alcohol.

b. Puede realizar cualquier tipo de ejercicio físico tras un periodo razonable de tiempo desde la intervención.

c. Es importante que el paciente lleve una dieta hiposódica, hipoglucémica e hiperlipídica.

d. No es necesario comunicar a la unidad de trasplante los síntomas leves de resfriado o gripe.

e. Se libera al paciente del control de peso y tensión arterial tras el trasplante.

f. Debe contactar con el equipo de trasplante siempre que se valla a vacunar.
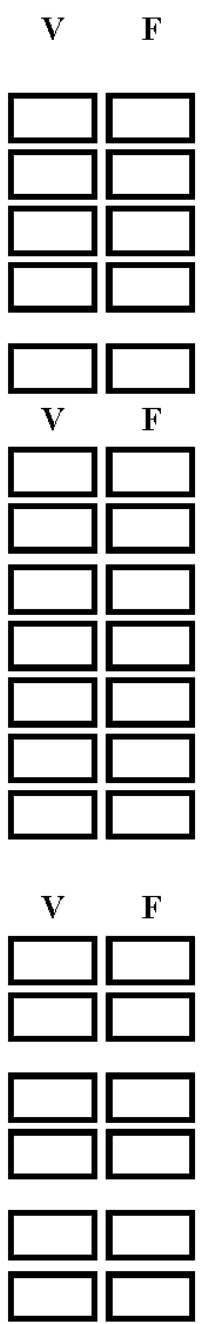


\section{RESULTADOS}

Los resultados del estudio se presentan en cuatro bloques que se corresponden con la estructura de la encuestas. Hemos establecido un criterio de selección para la elección de las preguntas a comentar donde aquellas con valores perdidos de más de un $40 \%$ han sido eliminadas, por no ser consideradas representativas, pero que si serán de utilidad para la elaboración del temario de las sesiones formativas que nos gustaría desarrollar.

De los 93 sujetos que forman la muestra han respondido 58 sujetos, lo que supone el $62 \%$ de la población (Figura 1).

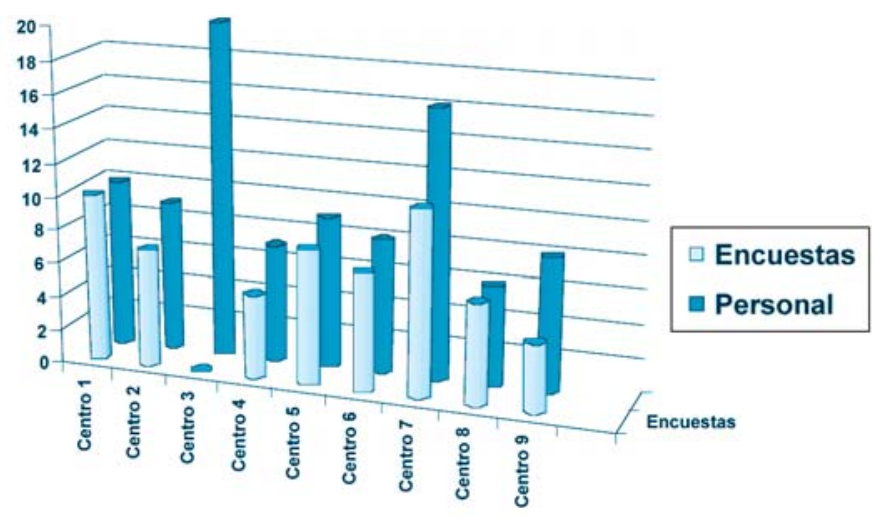

Figura 1. Personal y encuestas cumplimentadas por centro.

\section{Bloque 1}

En este bloque el primero de los aspectos que valoramos es el porcentaje de pacientes que demandan información sobre el trasplante renal al personal de enfermería de los centros periféricos (Tabla 1). Esta tabla refleja que el 55,6 $\%$ del personal de enfermería encuestado considera que menos del 50\% de los pacientes de cada centro les solicitan algún tipo de información sobre el trasplante renal. Mientras que solo el 7,4\% de los encuestados considera que todos los pacientes demandan información acerca del trasplante renal.

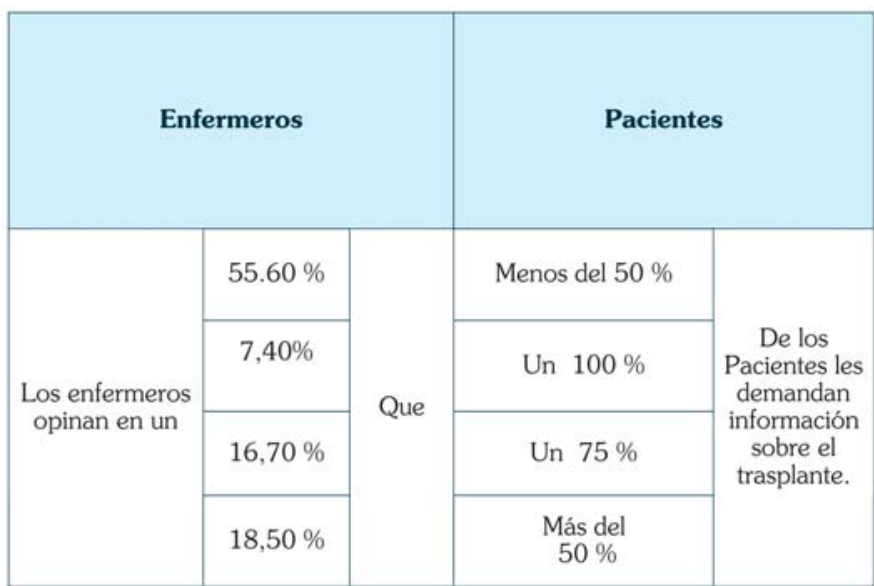

Tabla 1. Opinión del personal de enfermería en cuanto al porcentaje de pacientes que demandan información sobre el trasplante renal.
A su vez, el personal de enfermería señala como prioridad para los pacientes (Figura 2), recibir información en primer lugar sobre la lista de espera, seguida de la intervención quirúrgica y la preparación para el trasplante. En los últimos lugares sitúan la información referida a los autocuidados post trasplante, dietas y medicación tras el trasplante.

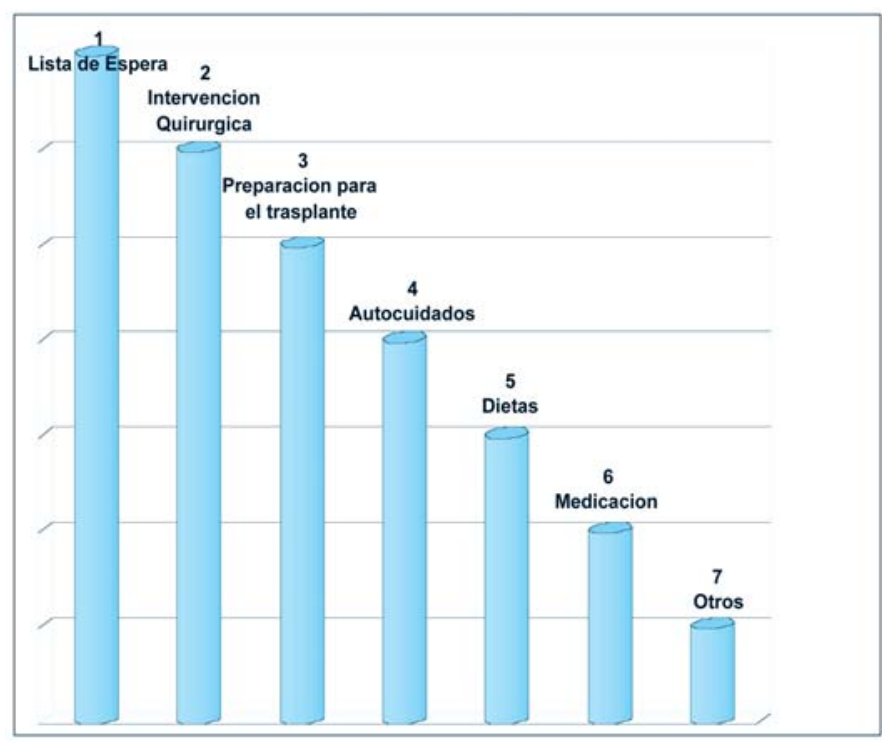

Figura 2. Prioridad en la demanda de información por parte de los pacientes según enfermería.

Cuando hemos preguntado acerca de la forma de dar la información hemos obtenidos los siguientes resultados (Figura 3). Uno de los ítems refleja que no existe un protocolo para proporcionar la información acerca del trasplante renal $(62,5 \%)$ frente a un $37,5 \%$ que afirma la existencia de este protocolo en su centro. Dicha información se da a demanda del paciente $(95,8 \%)$ o de forma espontánea por el personal de enfermería $(52,5 \%)$ sin llevar a cabo una programación de la misma (73\%). La información se da sin realizar una encuesta inicial para valorar las necesidades del paciente $(85,3 \%)$ y sin incluir a la familia $(66,7 \%)$. Al no existir un lugar destinado para dar dicha información $(97,1 \%)$, esta se transmite durante la sesión de hemodiálisis (86,3\%). El $74,3 \%$ de los enfermeros/as encuestados, afirman que no se proporciona ningún tipo de información escrita como complemento a la verbal.

\section{Bloque 2.}

Hemos reflejado las repuestas respecto a los procedimientos que se le realizan al paciente tanto para entrar en la lista de espera como en el pre-trasplante inmediato, y a la información que este recibe (Tabla 2). Para la inclusión en la lista de espera, uno de los aspectos más importantes, hace referencia a las pruebas y determinaciones inmunológicas a las que son sometidos los pacientes. 


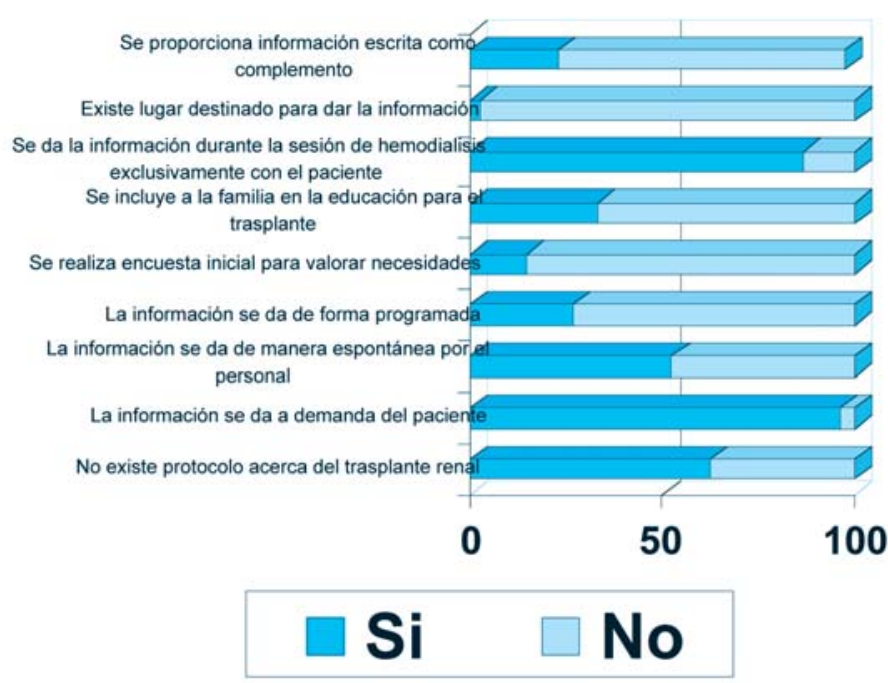

Figura 3. Forma de dar la información sobre el trasplante renal en los centros periféricos.

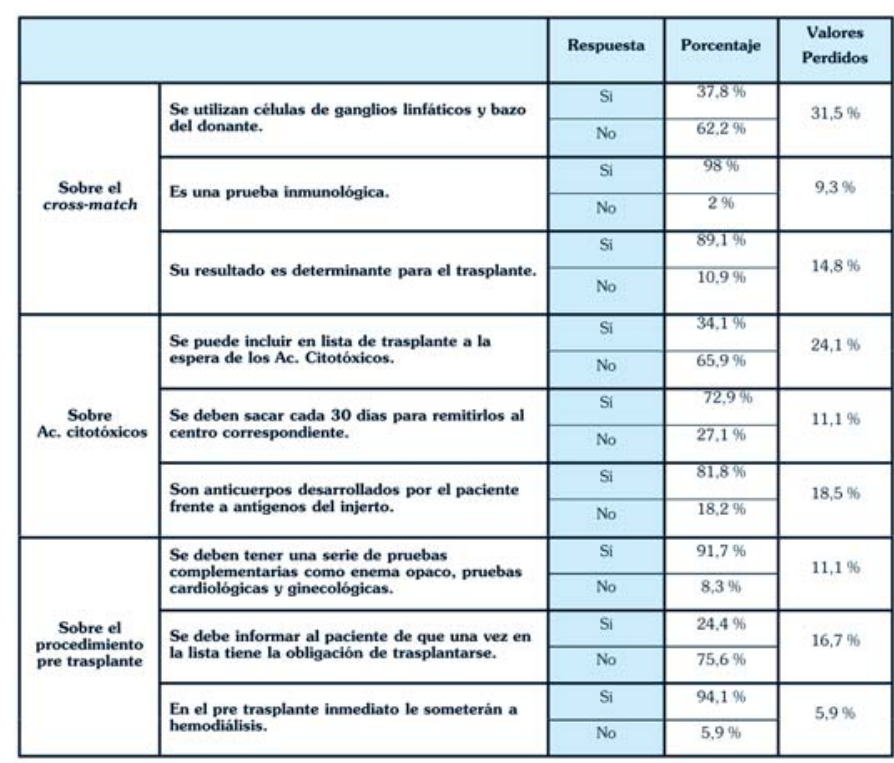

Tabla 2. Respuestas de los ítems más significativos respecto al pretrasplante renal.

Un 98\% de los encuestados identifican el cross-match como una prueba inmunológica pero no relaciona las células de los ganglios linfáticos o del bazo del donante como necesarios para dicha determinación (62,2\%). Hemos querido resaltar uno de los ítems en el que el $10,9 \%$ del personal de enfermería considera que el cross-match no es determinante para el trasplante mientras que un $89,1 \%$ afirma lo contrario.

El 81,8\% de los encuestados, reconocen la definición de los anticuerpos citotóxicos, y el 65,9\% de los mismos no incluiría en la lista de trasplante a un paciente sin haber obtenido el resultado de dichos anticuerpos.
Sin embargo, solo un $27,1 \%$ reconocen que los anticuerpos citotóxicos no se determinan cada 30 días (si no mediara una situación excepcional como transfusión o transplantectomía) frente a un $72,9 \%$ que afirman determinarlos cada mes, sin diferenciarlos de la extracción de la pruebas cruzadas que sí es mensual.

Los encuestados, en un $24,4 \%$ consideran que el paciente una vez que está en la lista de espera tiene la obligación de trasplantarse, sin embargo, un 91,7\%, reconoce la necesidad de tener una serie de pruebas complementarias para entrar en dicha lista, y un $94,1 \%$ considera la hemodiálisis como un paso previo necesario al trasplante renal.

\section{Bloque 3}

En este bloque, hemos querido resaltar los resultados obtenidos en los aspectos referentes a la estancia hospitalaria del paciente tras el trasplante y las preguntas referentes al nuevo órgano (Tabla 3).

\begin{tabular}{|c|c|c|c|c|}
\hline & & Respuesta & Porcentaje & $\begin{array}{l}\text { Valores } \\
\text { Perdidos }\end{array}$ \\
\hline \multirow{6}{*}{$\begin{array}{c}\text { Sobre el } \\
\text { protocolo } \\
\text { hospitalario del } \\
\text { trasplante renal }\end{array}$} & \multirow{2}{*}{$\begin{array}{l}\text { El paciente debe permanecer en la U.C.I. un } \\
\text { tiempo minimo de } 24 \text { horas. }\end{array}$} & $\mathrm{Si}$ & $95,8 \%$ & \multirow{2}{*}{$11,1 \%$} \\
\hline & & No & $4,2 \%$ & \\
\hline & \multirow{2}{*}{$\begin{array}{l}\text { Es imprescindible el uso de batas, gorros, } \\
\text { mascarillas y papis por los familiares durante } \\
\text { el tiempo de aislamiento. }\end{array}$} & $\mathrm{Si}$ & $92,6 \%$ & \multirow{2}{*}{$0 \%$} \\
\hline & & No & $7,4 \%$ & \\
\hline & \multirow{2}{*}{$\begin{array}{l}\text { El aislamiento en la unidad de trasplante se } \\
\text { mantendrá hasta el alta del paciente. }\end{array}$} & Si & $50 \%$ & \multirow{2}{*}{$11,1 \%$} \\
\hline & & No & $50 \%$ & \\
\hline \multirow{2}{*}{$\begin{array}{c}\text { Sobre las } \\
\text { complicaciones } \\
\text { del trasplante } \\
\text { renal }\end{array}$} & \multirow{2}{*}{$\begin{array}{l}\text { La complicación médica más frecuente tras el } \\
\text { trasplante es la hipertensión arterial. }\end{array}$} & Si & $47.5 \%$ & \multirow{2}{*}{$25,9 \%$} \\
\hline & & No & $52,5 \%$ & \\
\hline \multirow{8}{*}{$\begin{array}{l}\text { Sobre } \\
\text { el nuevo riñón. }\end{array}$} & \multirow{2}{*}{$\begin{array}{l}\text { La zona más frecuente de colocación del } \\
\text { riñón es la fosa iliaca o fosa lumbar. }\end{array}$} & Si & $96 \%$ & \multirow{2}{*}{$7.4 \%$} \\
\hline & & No & $4 \%$ & \\
\hline & \multirow{2}{*}{$\begin{array}{l}\text { En España todavia no existe la posibilidad de } \\
\text { donante vivo. }\end{array}$} & Si & $8.2 \%$ & \multirow{2}{*}{$9,3 \%$} \\
\hline & & No & $91.8 \%$ & \\
\hline & \multirow{2}{*}{$\begin{array}{l}\text { El tiempo de vida media del injerto suele ser } \\
\text { de } 20 \text { años. }\end{array}$} & Si & $37.8 \%$ & \multirow[t]{2}{*}{$16.7 \%$} \\
\hline & & No & $62,2 \%$ & \\
\hline & \multirow{2}{*}{$\begin{array}{l}\text { El paciente solo se puede trasplantar dos } \\
\text { veces. }\end{array}$} & Si & $6.7 \%$ & \multirow{2}{*}{$16,7 \%$} \\
\hline & & No & $93,3 \%$ & \\
\hline
\end{tabular}

Tabla 3. Respuestas de los ítems más significativos respecto a la estancia hospitalaria y al nuevo riñón.

Se encontró que en gran medida $(95,8 \%)$, el personal de enfermería conoce que el paciente permanecerá un mínimo de 24 horas en la UCI (según el protocolo vigente en nuestra provincia) tras el trasplante. En lo referente, a las medidas de aislamiento que se han de tomar con un paciente inmunodeprimido, como es el caso de un paciente trasplantado, el 92,6\% de los encuestados considera imprescindible el uso de batas, gorros, mascarillas y calzas por los familiares, mientras dure el periodo de aislamiento, considerando un $50 \%$ que este aislamiento se mantendrá hasta el alta del paciente.

Solo un $47,5 \%$ de los encuestados, reconoce la hipertensión arterial como la complicación médica más frecuente tras el trasplante renal, frente a un 52,5\% que ha optado por otras respuestas como la poliglobulia, la anemia, la diabetes esteroidea o el aumento de los niveles de i.P.T.H. 
En el apartado referente al nuevo riñón, como observamos en la tabla, el $96 \%$ de los sujetos identifica la fosa ilíaca o la fosa lumbar como los lugares más frecuentes de colocación del órgano a trasplantar. Como respuesta a la pregunta de si un paciente se puede trasplantar solo dos veces el 93,3\% ha respondido que esta afirmación es incorrecta, sin embargo al cuestionar acerca de la vida media del órgano trasplantado, en nuestro caso el riñón, un $37,8 \%$ afirma que es de 20 años.

Por último nos parece importante resaltar que sobre la posibilidad del trasplante de un donante vivo, un $8,2 \%$ no conoce la existencia de este tipo de trasplante en España.

\section{Bloque 4}

En cuanto a los datos obtenidos en el bloque referido a la etapa post trasplante renal (Tabla 4), observamos que se identifica este periodo como más indicado para el embarazo $(80,4 \%)$ que el periodo de dialisis $(19,6 \%)$, mejorando el trasplante renal las disfunciones sexuales $(95,7 \%)$ y reanudándose la menstruación y la ovulación en las mujeres que la hubieran podido perder en el periodo dialítico $(97,6 \%)$.

\begin{tabular}{|c|c|c|c|}
\hline & Respuesta & Porcentaje & $\begin{array}{l}\text { Valores } \\
\text { Perdidos }\end{array}$ \\
\hline \multirow{2}{*}{$\begin{array}{l}\text { Es preferible el embarazo en el periodo de dialisis que tras el } \\
\text { trasplante. }\end{array}$} & Si & $19,6 \%$ & \multirow{2}{*}{$14,8 \%$} \\
\hline & No & $80.4 \%$ & \\
\hline \multirow{2}{*}{$\begin{array}{l}\text { E embarazo es habitualmente más corto que en el resto de las } \\
\text { mujeres. }\end{array}$} & Si & $35 \%$ & \multirow{2}{*}{$25.9 \%$} \\
\hline & No & $65 \%$ & \\
\hline \multirow[b]{2}{*}{ El trasplante renal mejora sensiblemente las disfunciones sexuales. } & Si & $95.7 \%$ & \multirow{2}{*}{$14,8 \%$} \\
\hline & № & $4,3 \%$ & \\
\hline \multirow{2}{*}{$\begin{array}{l}\text { En la mujer trasplantada la menstruación y la ovulación pueden } \\
\text { reanudarse. }\end{array}$} & Si & $97,6 \%$ & \multirow{2}{*}{$22.2 \%$} \\
\hline & No & $2,4 \%$ & \\
\hline \multirow{2}{*}{$\begin{array}{l}\text { Puede realizar cualquier tipo de ejercicio fisico tras un periodo } \\
\text { razonable de tiempo desde la intervención. }\end{array}$} & Si & $54,9 \%$ & \multirow{2}{*}{$5,6 \%$} \\
\hline & № & $45,1 \%$ & \\
\hline \multirow{2}{*}{$\begin{array}{l}\text { Es importante que el paciente lleve una dieta hiposódica, } \\
\text { hipoglucémica e hiperlipidica. }\end{array}$} & Si & $49 \%$ & \multirow{2}{*}{9,39} \\
\hline & No & $51 \%$ & \\
\hline \multirow{2}{*}{$\begin{array}{l}\text { Se libera al paciente del control de peso y de la tensión arterial tras } \\
\text { el trasplante. }\end{array}$} & ST & $8,2 \%$ & \multirow{2}{*}{$9,3 \%$} \\
\hline & No & $91.8 \%$ & \\
\hline
\end{tabular}

Tabla 4. Respuesta de los ítems más significativos respecto a los autocuidados post trasplante.

El personal de enfermería de los centros periféricos, en un $91,8 \%$ de los casos, considera que el paciente debe continuar con los controles de peso y tensión arterial frente a un $8,2 \%$ que considera que se puede liberar al paciente de estas medidas.

Respecto al tipo de dieta que debe llevar un paciente renal han respondido correctamente un 51\% de los encuestados, además un $54,9 \%$ ha reconocido la posibilidad de realizar cualquier tipo de ejercicio físico tras un periodo de tiempo razonable tras el trasplante.

\section{DISCUSIÓN}

La formación continuada es una de las principales funciones que se promueve en los centros periféricos de hemodiálisis. Enfermería debe estar al día de cualquier posibilidad de tratamiento que pudiera afectar a los pacientes con los que cuida. En el caso que nos ocupa, la formación continuada sobre el trasplante renal, debería incluirse en el proceso de aprendizaje y formación del personal de enfermería de dichos centros, ya que como se refleja en el estudio de Rosa Ortelis sobre la actitud de la población laboral hospitalaria ante la generación de órganos ${ }^{(6)}$, una de las conclusiones es que el cuidado de pacientes con patologías susceptibles de trasplante en otros centros, no presupone conocimientos referentes a los trasplantes en general.

Pensamos que se podría mejorar la forma en que se da la información al paciente en los centros periféricos de diálisis, programando esta formación y basándola en los protocolos que existen en los centros. De la misma manera, deberíamos aportar esta información por escrito al paciente.

Con relación a la etapa del pre trasplante renal, que es sobre la que mayor información demandan los pacientes, deberíamos insistir en la necesidad de conocer las diferentes pruebas que se le deben realizar al paciente, previas al trasplante, como los anticuerpos citotóxicos o el cross-match; sin embargo, los conocimientos referidos a las contraindicaciones para el trasplante o a las técnicas inmediatas al mismo, son más completos. En cuanto al proceso del trasplante renal y la estancia hospitalaria del paciente, los resultados obtenidos nos muestran que los enfermeros/as de los centros periféricos conocen las actuaciones que se han de llevar a cabo en este periodo, aunque por estar fuera del ámbito hospitalario, existe una necesidad de actualizar dichos $\mathrm{co}^{-}$ nocimientos.

Por último, pensamos que podemos educar correctamente al paciente en lo relacionado con los autocuidados post-trasplante, aunque en este apartado no se ha profundizado, por ser este un proceso que se lleva a cabo en la unidad de trasplante, donde se debe insistir en aspectos como el manejo de la medicación y el estilo de vida de estos pacientes.

Concluimos que la mayor parte del personal de enfermería de los centros periféricos tiene amplia informa- 
ción sobre el trasplante renal, aunque en algunos aspectos, esta información debería completarse o actualizarse mediante sesiones formativas, las cuales nos proponemos organizar para una mejor atención de enfermería al paciente en dialisis en los centros periféricos.

\section{AGRADECIMIENTOS}

A los centros de dialisis Aljarafe, Montequinto, Sierra Este, Virgen de los Reyes, Santa Isabel, Bellavista, y a los centros periféricos de los hospitales Virgen de la Macarena y Virgen del Rocío, que han colaborado en la realización de este estudio por la atención e interés que ha prestado el personal de enfermería, ya que sin su colaboración este estudio no se podría haber llevado a cabo. A los coordinadores que nos han atendido y en muchos casos han colaborado en la recogida y resolución de dudas acerca de las encuestas.

A Patricia Villaciervos Moreno, por su inestimable colaboración y asesoramiento estadístico.

En especial a nuestro compañero Jesús Lucas Martín Espejo, por promover la realización de este trabajo.

\section{BIBLIOGRAFÍA}

1. Andreu L, Force E. 500 cuestiones que plantea el cuidado del enfermo renal. $2^{a}$ ed. Barcelona: Masson; 2001.
2. Oto Royo A, Muñoz Sancho R. Calidad de vida en los pacientes en hemodiálisis: influencias del estado de ansiedad-depresión y de otros factores de comorbilidad. En: Comunicaciones presentadas al XXVIII Congreso Nacional de la Sociedad Española de Enfermería Nefrológica. Palma de Mallorca 2003. Barcelona: Hospal; 2003.

3. Llach F, Valderrabano F. Insuficiencia renal crónica: Diálisis y trasplante renal. $2^{\mathrm{a}}$ ed. Volumen II. Madrid: Ediciones Norma; 1997.

4. Davidoff L. Introducción a la psicología. $3^{\text {a }}$ ed. México: Mc.Graw-Hill; 1995.

5. Varios Autores. Curso de experto universitario en enfermería nefrológica. Universitas Malacitana. Málaga. 2000. 325-338.

6. Ortelis R, Martín M.J, Soriano F, Fernández, M.C, Sainz, M.M, Cebollada M.A, et al. Actitud de la población laboral hospitalaria ante la generación de órganos. En: Comunicaciones presentadas al Congreso Nacional de la Sociedad Española de Enfermería Nefrológica. Bilbao1990. Barcelona: Hospal; 1990.

7. Puga M $\mathrm{M}^{\mathrm{a}} \mathrm{J}$, Rochera A, Berlango J. Protocolos de atención de enfermería a pacientes con trasplante renal. En: Manual de protocolos y procedimientos de actuación de enfermería nefrológica. Madrid: Janssen-Cilag; 2001.

8. Andres Casamiquela J, Fortuny I, Ventura C. Cuidados de enfermería en la insuficiencia renal. Madrid: Gallery/Health; 1993.

9. Escobar M $M^{a} \mathrm{~J}$, Velasco A. Protocolos de enfermería en la unidad de trasplante renal. Actualizaciones en Trasplante 2001. Hospital Universitario Virgen del Rocío. Sevilla. 2001. 